\title{
Fatores associados ao uso dos serviços de saúde bucal entre idosos no Brasil: scoping review
}

The use of oral health services by Brazilian elderly and associated factors: scoping review

Factores asociados al uso de los servicios de salud bucodental entre las personas mayores en Brasil: scoping review

Aline Soares Figueiredo Santos ORCID: https://orcid.org/0000-0002-3244-2378 Universidade Estadual de Montes Claros, Brasil E-mail: aline.santos@unimontes.br

Renata Francine Rodrigues Lima ORCID: https://orcid.org/0000-0002-7393-0415 Universidade Estadual de Montes Claros, Brasil E-mail: renata.oliveira@unimontes.br Simone de Melo Costa ORCID: https://orcid.org/0000-0002-0266-018X Universidade Estadual de Montes Claros, Brasil

E-mail: smelocosta@gmail.com

Mânia de Quadros Coelho ORCID: https://orcid.org/0000-0002-6913-8695 Universidade Estadual de Montes Claros, Brasil

E-mail: mania.coelho@unimontes.br

Cássia Pérola dos Anjos Braga Pires ORCID: https://orcid.org/0000-0002-8649-4675 Universidade Estadual de Montes Claros, Brasil

E-mail: peroladab@gmail.com

Paula Karolina Soares Farias ORCID: https://orcid.org/0000-0003-0529-2754 Universidade Estadual de Montes Claros, Brasil

E-mail: paulak.soares@hotmail.com

Maisson Santiago Soares Costa ORCID: https://orcid.org/0000-0003-1209-5660 Universidade Estadual de Montes Claros, Brasil E-mail: costamaisson@gmail.com Samuel Trezena

ORCID: https://orcid.org/0000-0002-4217-1276 Universidade Estadual de Montes Claros, Brasil E-mail: samueltrezena@gmail.com

Anna Paula Silva Dias

ORCID: https://orcid.org/0000-0003-1404-8171 Universidade Estadual de Montes Claros, Brasil E-mail: annapauladias8@gmail.com

Amanda Neves Magalhães ORCID: https://orcid.org/0000-0003-0516-6475 Universidade Estadual de Montes Claros, Brasil E-mail: amandanevesmag@gmail.com

Raquel Conceição Ferreira

ORCID: https://orcid.org/0000-0001-8897-9345

Universidade Federal de Minas Gerais, Brasil

E-mail: raquelconceicaoferreira@gmail.com

Marise Fagundes Silveira

ORCID: https://orcid.org/0000-0002-8821-3160 Universidade Estadual de Montes Claros, Brasil E-mail: marise.silveira@unimontes.br

Andrea Maria Eleutério de Barros Lima Martins ORCID: https://orcid.org/0000-0002-1205-9910 Universidade Estadual de Montes Claros, Brasil E-mail: martins.andreamebl@gmail.com 


\begin{abstract}
Resumo
Objetivo: Mapear e sintetizar evidências de pesquisas sobre fatores associados ao uso dos serviços de saúde bucal entre idosos brasileiros. Metodologia: Trata-se de uma scoping review guiada pela pergunta norteadora "Quais fatores estão associados ao uso dos serviços de saúde bucal pelos idosos brasileiros?". A busca dos artigos considerou a estratégia Population, Concept e Context (PCC), pela combinação de termos indexados e foi realizada nas bases de dados Medline/PubMed, Lilacs e SciELO, de maio a julho de 2019. Fundamentou-se a discussão pela teoria de Andersen \& Newman. Resultados: Dos 337 artigos identificados, 12 estudos transversais, publicados de 2007 a 2019 , atenderam aos critérios de inclusão. Os fatores associados ao uso de serviços de saúde bucal foram: idade, sexo, estado civil, escolaridade, etnia/cor/raça, conhecimento sobre saúde, renda, plano de saúde, região do país, zona urbana/rural, sintomas, diagnóstico da condição de saúde bucal e comportamento em saúde. Escolaridade, renda e diagnóstico da condição bucal foram os fatores mais evidenciados nos estudos. Conclusão: Diferentes fatores foram associados à utilização de serviços de saúde bucal por idosos brasileiros, com maior destaque aos sociodemográficos e de necessidade clínica. Identificar barreiras de acesso desigual aos serviços odontológicos poderá contribuir na redução do quadro de doenças bucais entre idosos, com necessidade acumulada de tratamento e reabilitação.
\end{abstract}

Palavras-chave: Idosos; Saúde bucal; Serviços de saúde bucal.

\begin{abstract}
Aim: To map and synthesize evidences on factors associated with the use of oral health services among Brazilian elderly. Methodology: This is a scoping review guided by the guiding question "What factors are associated with the use of oral health services by the elderly in Brazil?". The search for articles considered the Population, Concept and Context (PCC) strategy, by combining indexed terms and was carried out in the Medline/PubMed, Lilacs and SciELO databases, from May to July 2019. The discussion was based on theory by Andersen \& Newman. Results: A total of 337 articles were identified, 12 met the review inclusion criteria. Studies are cross-sectional, published between 2007 and 2019. Factors associated with the use of oral health services were: age, gender, marital status, education, ethnicity / color / race, health knowledge, income, health insurance, region of the country - urban / rural area, symptoms, diagnosis of oral health condition and health behavior. Schooling, income and diagnosis of oral condition were the most evidenced factors in the studies. Conclusion: Different factors were associated with the use of oral health services by Brazilian elderly, with greater emphasis on sociodemographic and clinical need. Identifying barriers to unequal access to dental services may contribute to the reduction of oral disease among the elderly, with an accumulated need for treatment and rehabilitation.
\end{abstract}

Keywords: Aged; Oral health; Dental health services.

\title{
Resumen
}

Objetivo: mapear y sintetizar evidencias de investigación sobre factores asociados al uso de servicios de salud bucal entre ancianos brasileños. Metodología: Se trata de una scoping review guiada por la pregunta orientadora "¿Qué factores están asociados con el uso de los servicios de salud bucal por parte de los ancianos brasileños?". La búsqueda de artículos consideró la estrategia de Población, Concepto y Contexto (PCC), mediante la combinación de términos indexados y se realizó en las bases de datos Medline/PubMed, Lilacs y SciELO, de mayo a julio de 2019. La discusión se basó en la teoría de Andersen \& Newman. Resultados: De los 337 artículos identificados, 12 estudios transversales, publicados entre 2007 y 2019, cumplieron con los criterios de inclusión. Los factores asociados a la utilización de los servicios de salud bucal fueron: edad, sexo, estado civil, escolaridad, etnia/color/raza, conocimientos sobre salud, ingreso, plan de salud, región del país, zona urbana/rural, síntomas, diagnóstico de Estado de salud oral y comportamiento de salud. La educación, la renta y el diagnóstico de la condición oral fueron los factores más evidenciados en los estudios. Conclusión: Diferentes factores se asociaron con el uso de los servicios de salud bucal por ancianos brasileños, con mayor énfasis en la necesidad sociodemográfica y clínica. La identificación de barreras para el acceso desigual a los servicios dentales puede contribuir a la reducción de las enfermedades bucales entre los ancianos, con una necesidad acumulada de tratamiento y rehabilitación.

Palabras clave: Anciano; Salud bucal; Servicios de salud dental.

\section{Introdução}

O envelhecimento populacional é um quadro mundial caracterizado pelo aumento percentual de pessoas com mais de 65 anos que segundo projeções vão superar o número da população jovem (Mendes, 2012). No Brasil, em 1996, entre os idosos, 2,7\% deles estavam acima de 65 anos e em 2000, já eram 5,4\% da população, com previsão de ultrapassar o percentual de jovens e chegar a 19\%, em 2050, com 253 milhões de habitantes (Malta \& Silva Jr, 2013; Miranda et al., 2016). Nesse contexto, o Brasil passa por uma transição demográfica, caracterizada pela diminuição da taxa de mortalidade e aumento da expectativa de vida, com aumento do número de idosos na população, trazendo uma transição epidemiológica pelo padrão de morbimortalidade marcado pela predominância de doenças e condições crônicas. Assim, o sistema de saúde precisa se adaptar 
para o cuidado à população idosa. Assim, devem ser elaboradas políticas de saúde que incentivem a prevenção, o cuidado e a atenção integral à saúde dos idosos e que os considerem como recurso importante para a sociedade (Brasil, 2005; Veras, 2009).

No que se refere à utilização dos serviços de saúde bucal pelos idosos, no Brasil, historicamente, houve uma atenção odontológica marcada pela falta de acesso e exclusão social. Portanto, há uma agenda acumulada de necessidades em saúde bucal nesse estrato populacional. Por décadas, a atenção odontológica preventiva foi voltada para escolares, excluindo os demais ciclos de vida e relegando-os às ações pontuais, do tipo curativas ou mutiladoras (Sampaio \& Cruz, 2014).

Diversos estudos foram conduzidos com o intuito de identificar os fatores associados ao uso de serviços de saúde bucal pelos idosos brasileiros (Sória et al., 2019; Matos \& Lima-Costa, 2007). Entre eles, destacam-se os levantamentos nacionais, que investigaram essa população em 2003 e em 2010 (Brasil, 2004; Brasil 2012). Estudos nacionais constataram baixa prevalência de uso de serviços de saúde bucal, por idosos (Brasil, 2004; Brasil, 2012). Inquérito domiciliar, de base populacional, realizado no Brasil, em 2013, pelo Instituto Brasileiro de Geografia e Estatística (IBGE), em parceria com o Ministério da Saúde, mostrou que 28,9\% de idosos, com 60 anos ou mais, consultaram um dentista no ano anterior à coleta de dados (Stopa et al., 2017). A Pesquisa Nacional de Saúde Bucal, SB Brasil 2010, mostrou que apenas 30,4\% de idosos, de 65 a 74 anos, visitaram o dentista no último ano. Evidenciou ainda, a precariedade das condições de saúde bucal da população idosa, marcada pelo edentulismo, alta experiência de cárie, de doenças periodontais e de necessidade de próteses (Brasil, 2012).

A exploração da temática utilização dos serviços de saúde bucal tem sido fundamentada em diferentes modelos teóricos explicativos (Andersen \& Newman, 1973; Andersen \& Davidson, 2007). O modelo proposto por Andersen e Newman (1973) aborda três grupos de características, que explicam a propensão de utilização dos serviços odontológicos pelo indivíduo: 1. predisposição (características individuais e sociodemográficas), 2. facilitação (renda e fatores relativos ao uso de serviços) e 3. necessidade (relacionadas à autopercepção e necessidades de saúde). A utilização desse modelo em vários inquéritos brasileiros e internacionais apresenta grande impacto na avaliação sobre o uso dos serviços de saúde bucal, devido à aplicabilidade em diferentes estratos etários, além de agregar determinantes individuais e sociais aos fatores que são estudados (Pinto et al., 2012).

Nessa perspectiva, diferentes fatores podem explicar o uso de serviços de saúde bucal, por idosos. Sintetizar os achados da literatura, por meio da sumarização dos fatores associados à utilização dos serviços pelos idosos, pode contribuir para melhor compreensão da temática e subsidiar propostas de oportunização de acesso aos serviços de saúde bucal. Isso possibilitaria a manutenção de uma saúde bucal satisfatória ou o tratamento precoce de doenças instaladas, reduzindo o agravamento de condições de saúde bucal, que geram tratamentos complexos e mutiladores (Austregésilo et al., 2015). Dessa forma, objetivou-se sintetizar e mapear evidências de pesquisas sobre fatores associados ao uso dos serviços de saúde bucal entre idosos brasileiros.

\section{Metodologia}

Realizou-se um estudo de Scoping Review, conforme método proposto pelo Instituto Joanna Briggs (Peters et al., 2015). Também denominada de Revisão de Escopo, ela é uma metodologia indicada para sintetizar evidências de pesquisas, mapear a literatura existente em um determinado campo, identificar lacunas do conhecimento e fazer recomendações para pesquisas futuras. É de natureza complexa e heterogênea e conduzida quando uma revisão sistemática (Moher et al., 2009), por ser precisa, avaliando intervenções e desfechos não for a melhor indicação (Arksey \& O’Malley, 2005; Anderson et al., 2008; Peters et al., 2015).

A pergunta norteadora de pesquisa foi "Quais fatores estão associados ao uso dos serviços de saúde bucal por idosos brasileiros?" Utilizou-se a estratégia Population, Concept e Context (PCC) para scoping review (Peters et al., 2015; Godfrey et 
al., 2013), tendo sido considerados: P- idosos; C- uso de serviços de saúde bucal e C- fatores associados ao uso de serviços de saúde bucal.

A busca foi realizada nas bases de dados Medline/PubMed, Lilacs e SciELO, de maio a julho de 2019. A estratégia de busca foi definida pela combinação dos operadores boleanos e dos descritores relacionados a cada um dos componentes da estratégia PCC (Peters et al., 2015), presentes no Descritores em Ciências da Saúde (DeCS) e seus sinônimos no Medical Subject Headings (MeSH): aged, elderly, dental health services, dental care, comprehensive dental care, oral health, dental clinics, dental health surveys. Dessa forma, a estratégia de busca na base de dados Medline/PubMed ocorreu da seguinte maneira: $(((($ Aged[Title/Abstract]) OR Elderly[Title/Abstract])) AND (((Dental Health Services[Title/Abstract]) OR Dental Care[Title/Abstract]) OR Comprehensive Dental Care[Title/Abstract])) AND (((Oral Health[Title/Abstract]) OR Dental Clinics[Title/Abstract]) OR Dental Health Surveys[Title/Abstract]). Os filtros aplicados foram: estudos em humanos, idosos e artigos na língua inglesa e portuguesa. Nas outras bases de dados, utilizaram-se os operadores boleanos AND, OR, title/abstract para a realização da busca, que se deu segundo as possibilidades de buscas de tais plataformas, com o objetivo de obter o número máximo de estudos possíveis. A construção do fluxograma de estudos incluídos foi realizado de acordo com orientações Preferred Reporting Items for Systematic Review and Meta-Analysis Protocols (PRISMA P) (Moher et al., 2009).

Duas pesquisadoras trabalharam paralelamente na seleção dos estudos, que se deu em uma primeira etapa pela inclusão de estudos que tinham em seu título ou resumo o tema da estratégia PCC e exclusão dos repetidos nas diferentes bases de dados. Passou-se à leitura do texto completo e foram excluídos os estudos: descritivos, de revisão de literatura, os que não apresentavam a variável desfecho relacionada ao 'uso dos serviços de saúde bucal', estudos que incluíram outras faixas etárias, bem com população idosa de outros países.

Em seguida, os dados extraídos foram sumarizados e organizados em planilhas, posteriormente em tabelas para descrição de: autor(es), ano de publicação, local do estudo, ano de coleta, população (n), faixa etária, análise estatística, variável desfecho relacionada ao uso de serviços de saúde bucal e fatores associados estatisticamente a esse uso. Adotou-se o modelo teórico de Andersen \& Newman (1973), para organização dos fatores associados ao uso de serviços de saúde bucal, a partir das características de predisposição, de facilitação ou capacitantes e de necessidades em saúde (Andersen \& Newman, 1973). A discussão dos resultados também fundamentou-se no referido modelo teórico.

\section{Resultados}

No que se refere à busca e seleção dos artigos, encontraram-se 337 estudos. Desses, 312 foram excluídos, sendo: 21 por serem duplicados e 291 por não se adequarem aos critérios nos títulos e resumos. Entre os 25 que atenderam aos critérios de elegibilidade para leitura na íntegra, um total de 12 estudos foram incluídos na Scoping Review (Figura 1). 
Figura 1. Fluxograma da seleção dos estudos.
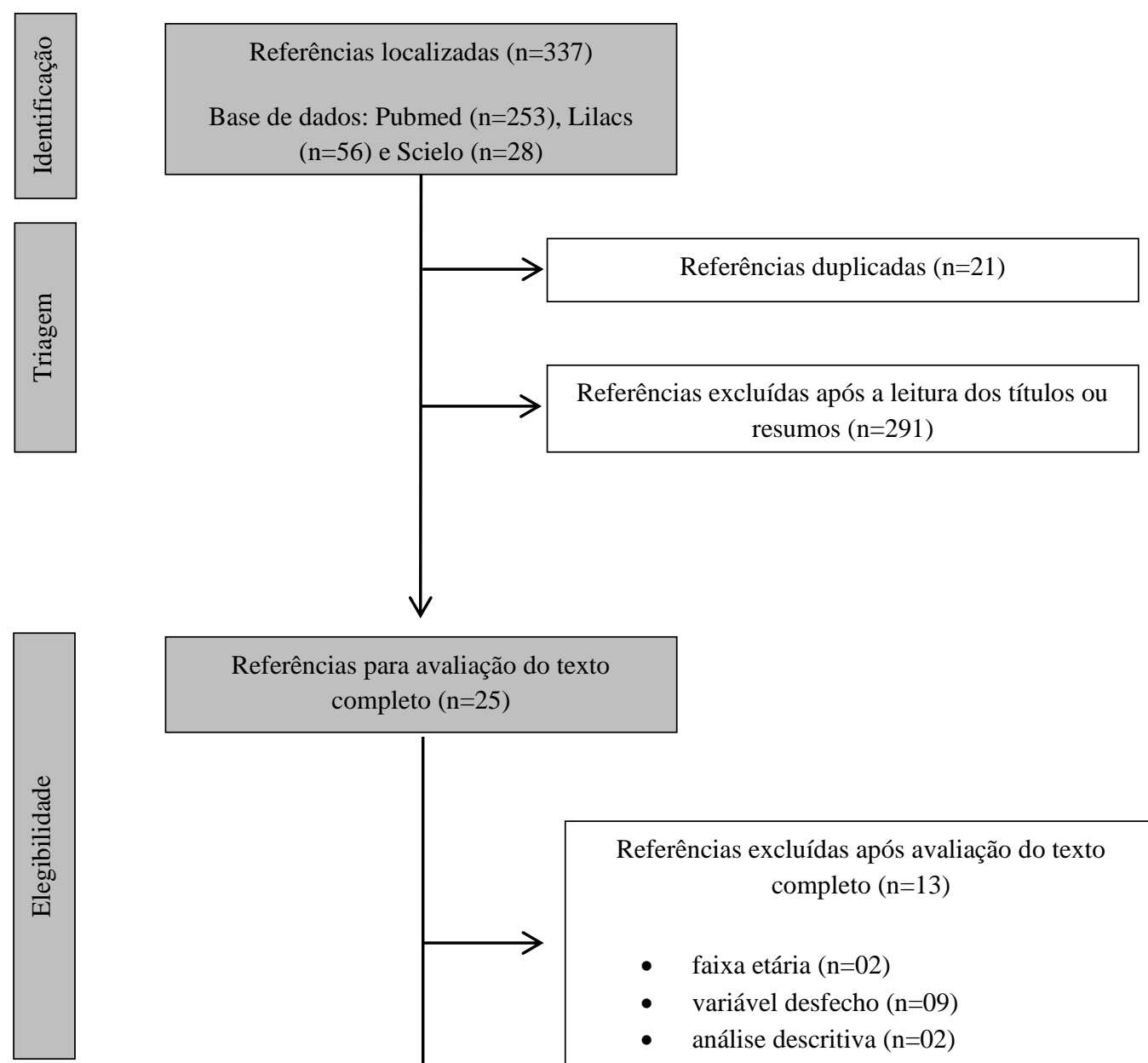

Referências para avaliação do texto completo $(n=25)$

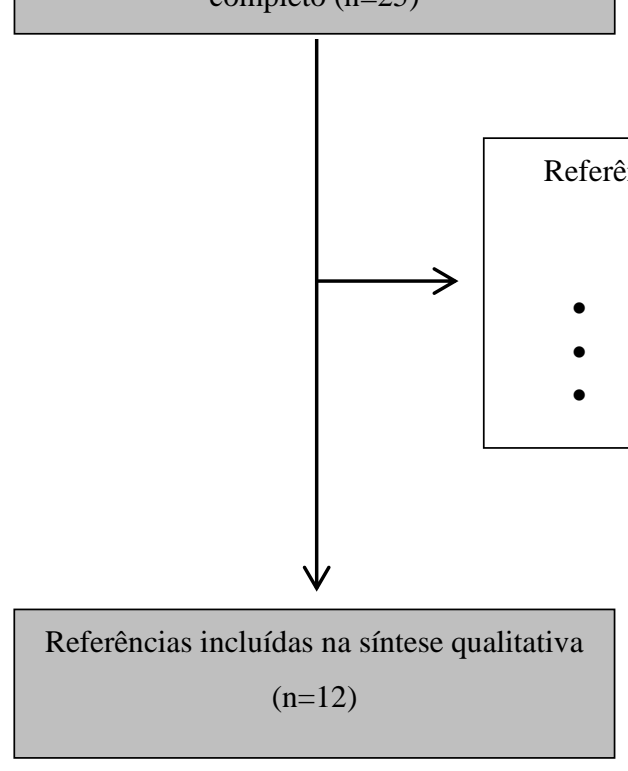

Fonte: Autores (2022).

Para todos os estudos houve cálculo amostral, com tamanho da amostra representativa da população, que oscilou entre 438 a 35.040 idosos, com idade igual e acima de 60 anos. O delineamento transversal foi adotado em todos os estudos. A análise estatística múltipla se deu através da técnica de regressão de Poisson (50,0\%) ((Sória et al., 2019; Silva et al., 2018; Prado et al., 2018; (Sória et al., 2019; Silva et al., 2018; Silva et al., 2013; Prado et al., 2018; Martins et al., 2008) e os demais 50,0\%, pela regressão logística (Fonseca et al., 2017; Oliveira et al., 2016; Souza et al., 2012; Martins et al., 2008; Martins et al., 2007; Matos \& Lima-Costa, 2007) (Tabela 1). 
Tabela 1 - Características dos estudos incluídos na revisão, por ordem cronológica decrescente de publicação (n=12). Brasil.

\begin{tabular}{|c|c|c|c|c|}
\hline Autores (Ano) & $\begin{array}{c}\text { Local do Estudo/Ano de } \\
\text { Coleta }\end{array}$ & População (n) & Faixa etária & Análise Estatística \\
\hline Sória et al. (2019) & $\begin{array}{c}\text { Pelotas }- \text { RS } \\
2014\end{array}$ & 1451 & $\geq 60$ anos & Regressão de Poisson \\
\hline Silva et al. (2018) & $\begin{array}{c}\text { Pelotas - RS } \\
\text { 2009-2010 }\end{array}$ & 438 & $\geq 60$ anos & Regressão de Poisson \\
\hline Prado et al. (2018) & $\begin{array}{c}\text { Brasil } \\
\text { Dados do SB Brasil } 2010\end{array}$ & 7619 & $65-74$ anos & Regressão de Poisson \\
\hline $\begin{array}{l}\text { Fonseca; Fonseca \& } \\
\text { Meneghim (2017) }\end{array}$ & $\begin{array}{c}\text { São Paulo } \\
\text { Dados do SB SP } 2015\end{array}$ & 5234 & $\geq 65$ anos & Regressão Logística Hierarquizada \\
\hline Oliveira et al. (2016) & $\begin{array}{c}\text { Montes Claros-MG } \\
\text { Dados SB Moc 2008-2009 }\end{array}$ & 480 & $65-74$ anos & Regressão Logística Hierarquizada \\
\hline $\begin{array}{l}\text { Silva; Langlois \& } \\
\text { Feldens (2013) }\end{array}$ & $\begin{array}{c}\text { Pelotas }- \text { RS } \\
\text { 2009-2010 }\end{array}$ & 438 & $\geq 60$ anos & Regressão de Poisson \\
\hline $\begin{array}{l}\text { Ferreira; Antunes \& } \\
\quad \text { Andrade (2013) }\end{array}$ & $\begin{array}{c}\text { Brasil } \\
\text { Dados do SB Brasil } 2010\end{array}$ & 6702 & $65-74$ anos & Regressão de Poisson \\
\hline Souza et al. (2012) & $\begin{array}{c}\text { Brasil } \\
\text { Dados do SB Brasil } \\
\text { 2002/2003 }\end{array}$ & 5108 & $65-74$ anos & Regressão Logística \\
\hline Martins et al. (2008) & $\begin{array}{c}\text { Brasil } \\
\text { Dados do SB Brasil 2002- } \\
2003\end{array}$ & 5009 & $65-74$ anos & $\begin{array}{c}\text { Regressão de Poisson com Estimação } \\
\text { da Variância Robusta }\end{array}$ \\
\hline $\begin{array}{l}\text { Martins; Barreto \& } \\
\text { Pordeus (2008) }\end{array}$ & $\begin{array}{l}\text { Região Sudeste do Brasil } \\
\text { Dados do SB Brasil 2002- } \\
2003\end{array}$ & 1014 & $65-74$ anos & Análise Logística Múltipla \\
\hline $\begin{array}{l}\text { Martins; Barreto \& } \\
\text { Pordeus (2007) }\end{array}$ & $\begin{array}{c}\text { Brasil } \\
\text { Dados do SB Brasil 2002- } \\
2003\end{array}$ & 5349 & $65-74$ anos & Regressão Logística \\
\hline \multirow{2}{*}{$\begin{array}{l}\text { Matos \& Lima-Costa } \\
\text { (2007) }\end{array}$} & $\begin{array}{c}\text { Brasil } \\
\text { PNAD* } 1998\end{array}$ & 28.943 & \multirow[t]{2}{*}{$\geq 60$ anos } & \multirow[t]{2}{*}{ Regressão Logística Multinomial } \\
\hline & $\begin{array}{c}\text { Brasil } \\
\text { PNAD* } 2003\end{array}$ & 35.040 & & \\
\hline
\end{tabular}

PNAD*: Pesquisa Nacional por amostra de domicílios. Fonte: Autores (2021).

As variáveis desfecho relacionadas ao uso de serviços de saúde bucal foram: tempo da última consulta odontológica, uso regular dos serviços, uso dos serviços por rotina e uso de serviços públicos. Os estudos que analisaram 'última consulta odontológica' adotaram diferentes recortes de tempo para análise dos fatores associados: uso há menos de um ano (Sória et al., 2019; Souza et al., 2012; Martins et al., 2008; Martins et al., 2007), entre um a dois anos (Prado et al., 2018; Ferreira et al. 2013; Matos \& Lima-Costa, 2007) e maior que três anos (Silva et al., 2013), bem como nunca utilizou (Matos \& Lima-Costa, 2007).

A maior escolaridade e a maior renda foram associadas ao menor tempo da última consulta odontológica $(<1$ ano) (Sória et al., 2019; Souza et al., 2012; Martins et al., 2008; Martins et al. 2007). Em contraposição, a maior escolaridade ficou associada à consulta há mais de três anos (Silva et al., 2013). Tanto para os idosos da região Sudeste (Martinset al., 2008) como de todo país (Martins et al., 2008), não ter necessidade de prótese dentária se associou à consulta no último ano, diferentemente dos idosos de Pelotas (RS) (Sória et al., 2019), com associação para aqueles com necessidade de prótese (Tabela 2). 
Tabela 2 - Tempo da última consulta odontológica entre idosos brasileiros e fatores associados (n=8). Brasil.

\begin{tabular}{|c|c|c|c|c|}
\hline Autor (es) /Ano & Variável desfecho & $\begin{array}{c}\text { Categoria de } \\
\text { análise }\end{array}$ & \multicolumn{2}{|c|}{$\begin{array}{c}\text { Fatores Associados com a categoria de análise } \\
\text { Análise Múltipla }(\mathbf{p}<0,05)\end{array}$} \\
\hline Sória et al. (2019) & $\begin{array}{l}\text { Última consulta } \\
\text { odontológica }\end{array}$ & $<1$ ano & $\begin{array}{l}\text { - 65-70 anos (idosos mais jovens) } \\
\text { - Ensino superior completo } \\
\text { - Com companheiro } \\
\text { - Relato de problemas bucais } \\
\text { - Necessidade de prótese } \\
\text { - Não edêntulos }\end{array}$ & \\
\hline Souza et al. (2012) & $\begin{array}{l}\text { Última consulta } \\
\text { odontológica }\end{array}$ & $<1$ ano & $\begin{array}{l}\text { - Brancos } \\
\text { - Dor dentária } \\
\text { - Renda > } 2 \text { salários mínimos } \\
\text { - Escolaridade > } 9 \text { anos }\end{array}$ & \\
\hline $\begin{array}{l}\text { Martins; Barreto \& } \\
\text { Pordeus (2008) }\end{array}$ & $\begin{array}{l}\text { Última consulta } \\
\text { odontológica }\end{array}$ & $<1$ ano & $\begin{array}{l}\underline{\text { Entre Dentados }} \\
\text { - Residentes na zona urbana } \\
\text { - Renda per capita } \geq 201,00 \text { reais } \\
\text { - Uso por rotina } \\
\text { - Sem necessidades de prótese } \\
\text { - Condição periodontal saudável } \\
\text { - Autopercepção ótima/ boa da } \\
\text { aparência } \\
\text { - Autopercepção ruim/ péssima da } \\
\text { fala }\end{array}$ & $\begin{array}{l}\text { Entre Edentados } \\
\text { - Escolaridade de } 5 \text { a } 8 \text { anos } \\
\text { - Uso por rotina } \\
\text { - Presença de dor } \\
\text { - Autoperceção do relacionamento } \\
\text { não afetado pela saúde bucal } \\
\text { - Autopercepção ruim/péssima da } \\
\text { aparência }\end{array}$ \\
\hline $\begin{array}{l}\text { Martins; Barreto \& } \\
\text { Pordeus (2007) }\end{array}$ & $\begin{array}{l}\text { Última consulta } \\
\text { odontológica }\end{array}$ & $<1$ ano & $\begin{array}{l}\text { Entre Dentados } \\
\text { - Escolaridade } \geq 5 \text { anos } \\
\text { - Acesso a informação de saúde } \\
\text { bucal } \\
\text { - Autopercepção ótima /boa da saúde } \\
\text { bucal } \\
\text {-Autopercepção } \\
\text { regular/ruim/péssima da mastigação } \\
\text { - Renda per capita } \geq \mathrm{R} \$ 201,00 \\
\text { - Dor em dentes ou gengivas } \\
\text { - Sem necessidades de prótese } \\
\text {-Sem necessidades de tratamento } \\
\text { periodontal } \\
\text { - Menos perda dentária }(0 \text { a } 15)\end{array}$ & $\begin{array}{l}\text { Entre Edentados } \\
\text { - Brancos } \\
\text { - Escolaridade } \geq 5 \text { anos } \\
\text { - Acesso à informação de saúde bucal } \\
\text { - Dor em dentes ou gengivas }\end{array}$ \\
\hline Prado et al. (2018) & $\begin{array}{l}\text { Última consulta } \\
\text { odontológica }\end{array}$ & $>1$ ano & $\begin{array}{l}\text { - Presença de sangramento e/ou cálculc } \\
\text { - Presença de bolsa periodontal } \\
\text { - Edentulismo }\end{array}$ & dental \\
\hline $\begin{array}{l}\text { Matos \& Lima- } \\
\text { Costa (2007) }\end{array}$ & $\begin{array}{l}\text { Última consulta } \\
\text { odontológica }\end{array}$ & & $\begin{array}{l}\frac{\text { Dados do PNAD* } 1998}{\text {-Idosos } \geq 65 \text { anos }} \\
\text { - Escolaridade } \leq 3 \text { anos } \\
\text { - Autopercepção da saúde geral } \\
\text { como regular/ruim/muito ruim } \\
\text { - Renda per capita } \leq \mathrm{R} \$ 130,00 \\
\text { - Sem plano privado de saúde } \\
\text { - Região Sudeste/ Nordeste em } \\
\text { relação ao Sul }\end{array}$ & $\begin{array}{l}\text { Dados do PNAD* } 2003 \\
\text { - Idosos } \geq 70 \text { anos } \\
\text { - Escolaridade } \leq 3 \text { anos } \\
\text { - Mulheres } \\
\text { - Autopercepção da saúde geral como } \\
\text { regular/ruim/muito ruim } \\
\text { - Renda per capita } \leq \mathrm{R} \$ 240,00 \\
\text { - Sem plano privado de saúde } \\
\text { - Região Sudeste/ Nordeste/ Centro } \\
\text { Oeste e Norte em relação ao Sul } \\
\text { - Residente na zona rural }\end{array}$ \\
\hline & & $\begin{array}{l}\text { Nunca utilizou } \\
\text { Grupos de } \\
\text { análises: } \\
\text { PNAD* } 1998 \\
\text { e } 2003\end{array}$ & $\begin{array}{l}\text { Dados do PNAD* } 1998 \\
\text { - Idosos } \geq 65 \text { anos } \\
\text { - Escolaridade } \leq 3 \text { anos } \\
\text { - Homens }\end{array}$ & $\begin{array}{l}\frac{\text { Dados do PNAD*2003 }}{\text { - Idosos } \geq 65 \text { anos }} \\
\text { - Escolaridade } \leq 3 \text { anos } \\
\text { - Homens } \\
\text { - Autopercepção da saúde geral como }\end{array}$ \\
\hline
\end{tabular}


- Autopercepção da saúde geral ruim/muito ruim

como ruim/muito ruim - Renda per capita $\leq \mathrm{R} \$ 250,00$

- Renda per capita $\leq \mathrm{R} \$ 130,00 \quad$ - Sem plano privado de saúde

- Sem plano privado de saúde — - Região Sudeste/ Nordeste em

- Região Sudeste/ Nordeste em relação ao Sul relação ao Sul

\begin{tabular}{|c|c|c|c|}
\hline $\begin{array}{l}\text { Ferreira; Antunes } \\
\& \text { Andrade (2013) }\end{array}$ & $\begin{array}{l}\text { Última consulta } \\
\text { odontológica }\end{array}$ & $\leq 2$ anos & $\begin{array}{l}\text { - Escolaridade } \geq 08 \text { anos } \\
\text { - Renda } \geq \mathrm{R} \$ 1501,00 \\
\text { - Residentes na região Sudeste do Brasil } \\
\text { - Com } 21 \text { dentes ou mais } \\
\text { - Com necessidade de tratamento odontológico } \\
\text { - Sem necessidade de próteses } \\
\text { - Usa próteses }\end{array}$ \\
\hline $\begin{array}{l}\text { Silva; Langlois \& } \\
\text { Feldens (2013) }\end{array}$ & $\begin{array}{l}\text { Última consulta } \\
\text { odontológica }\end{array}$ & $>3$ anos & $\begin{array}{l}\text { - Autoavaliação da saúde geral como ruim ou muito ruim } \\
\text { - Escolaridade > } 8 \text { anos } \\
\text { - Ex usuários de álcool } \\
\text { - Indivíduos edêntulos }\end{array}$ \\
\hline
\end{tabular}

PNAD*: Pesquisa Nacional por amostra de domicílios. Fonte: Autores (2021).

Investigaram-se ainda fatores associados ao uso regular (Silva et al., 2018), ao uso por rotina (Martins et al., 2008) e ao uso dos serviços públicos (Fonseca et al., 2017; Oliveira et al., 2016). Uso de serviço odontológico, de forma regular, foi associado à presença de pelo menos um dente, ou seja, idosos dentados (Silva et al., 2018). O uso de serviço odontológico por rotina teve associação com idosos residentes na zona urbana, ter acesso à informação em saúde bucal, não ter necessidade de prótese dentária e ter autopercepção que o relacionamento social não foi afetado pela condição de sua saúde bucal (Martins et al., 2008). Para uso do serviço público (Fonseca et al., 2017; Oliveira et al., 2016), o maior uso foi entre aqueles com menor escolaridade, menor renda, 'dor' como uma das motivações para uso do serviço e autopercepção ruim da condição de saúde bucal/aparência (Tabela 3). 
Tabela 3 - Fatores associados ao uso regular, uso por rotina e uso dos serviços públicos de saúde entre idosos brasileiros $(\mathrm{n}=$ 4). Brasil.

\begin{tabular}{|c|c|c|c|}
\hline Autores/Ano & Variável desfecho & $\begin{array}{l}\text { Categoria de } \\
\text { análise }\end{array}$ & $\begin{array}{c}\text { Fatores Associados com a categoria de análise } \\
\text { Análise Múltipla }(\mathbf{p}<0,05)\end{array}$ \\
\hline Silva et al. (2018) & Uso regular & Sim & - Presença de pelo menos um dente \\
\hline Martins et al. (2008) & Uso por rotina & Sim & $\begin{array}{l}\text { Entre Dentados } \\
\text { - Zona Urbana } \\
\text { - Acesso à informação de saúde } \\
\text { bucal } \\
\text { - Renda per capita } \geq 201,00 \text { reais } \\
\text { - Não ter necessidades de prótese } \\
\text { - Condição periodontal saudável } \\
\text { - Autopercepção ótima/ boa da } \\
\text { mastigação } \\
\text { - Autopercepção de } \\
\text { relacionamento social não afetado } \\
\text { pela saúde bucal } \\
\text { - Ausência de dor }\end{array}$ \\
\hline $\begin{array}{l}\text { Fonseca; Fonseca \& } \\
\text { Meneghim (2017) }\end{array}$ & $\begin{array}{l}\text { Uso de serviço } \\
\text { odontológico público }\end{array}$ & Maior & $\begin{array}{l}\text { - Menor escolaridade } \\
\text { - Não brancos } \\
\text { - Menor renda } \\
\text { - Motivo de uso: dor/extração } \\
\text { - Autopercepção negativa da condição bucal } \\
\text { - Idosos sem necessidade de prótese superior } \\
\text { - Idosos sem necessidade de prótese inferior }\end{array}$ \\
\hline Oliveira et al. (2016) & $\begin{array}{l}\text { Uso de serviço } \\
\text { odontológico público }\end{array}$ & Maior & $\begin{array}{l}\text { - Menor escolaridade } \\
\text { - Menor renda per capita } \\
\text { - Motivo de uso: sangramento, cavidade e dor } \\
\text { - Autopercepção negativa da aparência } \\
\text { - Relacionamento afetado pela saúde bucal } \\
\text { - Idosos que realizam o autoexame bucal } \\
\text { - Avaliação do atendimento ótimo/bom }\end{array}$ \\
\hline
\end{tabular}

Fonte: Autores (2021).

A Tabela 4 demonstra o mapeamento dos estudos a partir do modelo teórico sobre uso dos serviços de saúde, Andersen e Newman (1973). A escolaridade (predisposição de uso), a renda (como fator facilitador de uso) e o diagnóstico (relacionado à necessidade de uso) foram os fatores que mais frequentemente se associaram ao uso dos serviços de saúde bucal, entre os estudos (Tabela 4). 
Tabela 4 - Mapeamento dos estudos conforme associações com fatores que explicam o uso dos serviços de saúde bucal entre idosos brasileiros. Brasil.

\begin{tabular}{|c|c|}
\hline $\begin{array}{c}\text { Modelo teórico de } \\
\text { Andersen e Newman }\end{array}$ & Estudos \\
\hline \multicolumn{2}{|l|}{ PREDISPOSIÇÃO } \\
\hline \multicolumn{2}{|c|}{ Características Demográficas } \\
\hline Idade & Sória et al. (2019), Matos \& Lima-Costa (2007) \\
\hline Sexo & Matos \& Lima-Costa (2007) \\
\hline Estado Civil & Sória et al. (2019) \\
\hline \multicolumn{2}{|c|}{ Características de Estrutura Social } \\
\hline Escolaridade & $\begin{array}{l}\text { Sória et al. (2019), Souza et al. (2012), Martins; Barreto \& Pordeus (2008), Martins; Barreto \& Pordeus (2007), } \\
\text { Matos \& Lima-Costa (2007), Ferreira; Antunes \& Andrade (2013), Silva; Langlois \& Feldens (2013), Fonseca; } \\
\text { Fonseca \& Meneghim (2017), Oliveira et al. (2016), Martins et al. (2008) }\end{array}$ \\
\hline Etnia & $\begin{array}{l}\text { Souza et al. (2012), Martins; Barreto \& Pordeus (2007), Fonseca; Fonseca \& Meneghim (2017), Martins et al. } \\
\text { (2008) }\end{array}$ \\
\hline \multicolumn{2}{|l|}{ Características de } \\
\hline $\begin{array}{l}\text { Conhecimento sobre a } \\
\text { saúde }\end{array}$ & Martins et al. (2008), Martins; Barreto \& Pordeus (2007) \\
\hline \multicolumn{2}{|l|}{ FACILITAÇÃO } \\
\hline \multicolumn{2}{|l|}{$\begin{array}{l}\text { Características } \\
\text { familiares }\end{array}$} \\
\hline Renda & $\begin{array}{l}\text { Souza et al. (2012), Martins; Barreto \& Pordeus (2008), Martins; Barreto \& Pordeus (2007), Matos \& Lima- } \\
\text { Costa (2007), Ferreira; Antunes \& Andrade (2013), Fonseca; Fonseca \& Meneghim (2017), Oliveira et al. } \\
\text { (2016), Martins et al. (2008) }\end{array}$ \\
\hline Plano ou seguro de Saúde & Matos \& Lima-Costa (2007), Martins et al. (2008) \\
\hline \multicolumn{2}{|l|}{$\begin{array}{l}\text { Características da } \\
\text { comunidade }\end{array}$} \\
\hline Região do país & Matos \&Lima-Costa (2007), Ferreira; Antunes \& Andrade (2013) \\
\hline Zona urbana ou rural & Martins; Barreto \& Pordeus (2008), Matos \& Lima-Costa (2007), Martins et al. (2008) \\
\hline \multicolumn{2}{|l|}{ NECESSIDADE } \\
\hline Sintomas & $\begin{array}{l}\text { Sória et al. (2019), Souza et al. (2012), Martins et al. (2008), Martins; Barreto \& Pordeus (2008), Martins; } \\
\text { Barreto \& Pordeus (2007) }\end{array}$ \\
\hline Diagnóstico & $\begin{array}{l}\text { Sória et al. (2019), Martins; Barreto \& Pordeus (2008), Martins; Barreto \& Pordeus (2007), Prado et al. (2018), } \\
\text { Ferreira; Antunes \& Andrade (2013), Silva; Langlois \& Feldens (2013), Silva et al. (2018), Fonseca; Fonseca \& } \\
\text { Meneghim (2017), Martins et al. (2008) }\end{array}$ \\
\hline $\begin{array}{l}\text { Motivo para o cuidado - } \\
\text { comportamento }\end{array}$ & Martins; Barreto \& Pordeus (2008), Fonseca; Fonseca \& Meneghim (2017), Oliveira et al. (2016) \\
\hline
\end{tabular}

Fonte: Autoras (2021).

\section{Discussão}

O modelo de uso de serviços proposto por Andersen e Newman (1973) tem sido bastante utilizado para avaliar os fatores que interferem no uso de serviços médicos e também odontológicos nos últimos anos (Andersen \& Newman (1973; Andersen, 1995; Andersen; Davidson, 1997; Davidson \& Andersen, 1997; Andersen \& Davidson, 2007). O mapeamento realizado nesta Scoping Review mostrou que os fatores associados significativamente ao uso dos serviços de saúde bucal pelos idosos brasileiros se enquadram na abordagem de Andersen e Newman (1973).

As características de predisposição de uso dos serviços de saúde bucal entre idosos brasileiros foram idade (Sória et al., 2019; Matos \& Lima-Costa, 2007), sexo (Matos \& Lima-Costa, 2007) e estado civil (Matos \& Lima-Costa, 2007). A idade foi associada ao tempo da última consulta odontológica, sendo que os idosos mais jovens utilizaram mais recentemente os serviços de saúde bucal (Sória et al., 2019; Matos \& Lima-Costa, 2007). O aumento da idade foi associado com o fato de nunca ter usado os serviços ou ter usado há mais de um ano (Sória et al., 2019; Matos \& Lima-Costa, 2007). Corroborando 
com esse achado, Baldani et al. (2010), em Ponta Grossa, Paraná, encontraram que 67\% dos idosos não usavam o referido serviço há três anos ou mais (Baldani et al., 2010). A idade mais avançada e o comportamento de visitar menos ou não visitar o dentista provavelmente devem-se ao estilo de vida, à falta de dentes naturais e às questões culturais de um estrato etário marcado pela falta de acesso e oportunidade de cuidados com a saúde bucal. Com relação ao sexo, Matos \& Lima-Costa (2007) encontraram associações positivas entre sexo feminino e uso mais recente e, em contraposição, o sexo masculino se associou ao fato de nunca ter usado os serviços de saúde bucal. Uma provável explicação é o fato de as mulheres serem mais cuidadosas com a saúde. O estudo de Pelotas (Sória et al., 2019) encontrou que idosos com companheiros tiveram mais chances de usar o serviço há menos de um ano. Em um estudo realizado em indivíduos maiores de 55 anos na Austrália, não apresentar companheiro apresentou associação com a menor procura aos serviços de saúde bucal (Mariño et al., 2014). Estudos europeus mostraram que idosos com companheiros podem se sentir estimulados a se cuidarem, bem como terem ajuda para procurarem serviços de cuidados com a saúde (Holm-Pedersen et al., 2005; Listl, 2011; Tsakos et al., 2013).

Ainda como características de predisposição, os fatores relativos à estrutura social, como escolaridade e etnia, foram associados ao uso de serviços de saúde bucal entre idosos. A maior escolaridade foi associada ao uso mais recente, no último ano (Sória et al., 2019; Souza et al., 2012; Martins et al. 2008; Martins et al., 2007), ou menor que dois anos (Ferreira; Antunes \& Andrade, 2013), ou uso por rotina (Martins et al., 2008); enquanto a menor escolaridade foi associada ao uso maior que três anos ou nunca ter usado (Silva et al., 2013; Matos \& Lima-Costa, 2007). Machado et al. (2012) encontraram para idosos, em áreas de vulnerabilidade social, em Porto Alegre, RS, que a população com maior escolaridade tende a buscar mais regularmente os serviços de saúde bucal. Muito provavelmente pela maior probabilidade de acesso às informações (Machado et al., 2012). Nessa revisão, a menor escolaridade foi associada ao maior uso dos serviços públicos, pela população de Montes Claros - MG e de São Paulo - SP, sugerindo equidade na utilização dos serviços do Sistema Único de Saúde (SUS) (Fonseca et al., 2017; Oliveira et al., 2016), sendo que essa informação pode estar associada a variável renda, uma vez que idosos que apresentam maior escolaridade podem apresentar maior poder aquisitivo e assim buscar serviços de saúde bucal privados.

Como última característica de predisposição, a raça/cor/etnia autodeclarada foi analisada em três estudos com amostra representativa da população brasileira, com dados do SB Brasil 2002-2003. Os idosos de cor branca apresentaram maior chance de utilizar os serviços de saúde bucal há menos de um ano (Souza et al., 2012; Martins et al., 2007) e por rotina (Martins et al., 2008). No estudo de Fonseca et al. (2017), com dados da Pesquisa de Saúde Bucal do estado de São Paulo, SB SP 2015, para uso de serviços públicos, a chance de utilização foi maior entre não-brancos. Souza et al. (2012) mostraram resultados de menor chance de um idoso negro acessar os serviços que o idoso branco. Como característica de predisposição de uso dos serviços de saúde, o acesso às informações em saúde bucal esteve associado ao uso no último ano e por rotina. Inferese a partir daí que os alfabetizados em saúde se encontram mais orientados para utilizar os serviços, com mais regularidade (Martins et al., 2008; Martins et al., 2007).

Dentre os fatores de facilitação do uso dos serviços de saúde, as características familiares se associaram à maior renda, em estudos que avaliaram o uso recente (Ferreira et al., 2013; Souza et al., 2012; Martins et al., 2008; Martins et al. 2007; Matos \& Lima-Costa, 2007) ou por rotina (Martins et al., 2008). Moreira et al. (2005), em revisão sistemática, concluíram que a baixa renda constitui uma das principais barreiras para o uso de serviços de saúde bucal entre idosos. Nesta revisão, idosos com menor renda (Fonseca et al., 2017; Oliveira et al., 2016) tiveram maior chance de utilizar o serviço público, provavelmente pela baixa capacidade de desembolso direto para pagamento de serviços odontológicos. O uso dos serviços privados de saúde bucal foi associado com o uso por rotina entre idosos brasileiros edentados (Martins et al., 2008). Estudo com dados da PNAD de 1998 e de 2003 associou a ausência de um plano privado de saúde com o uso há mais de um ano ou com o fato de nunca ter usado os serviços de saúde bucal (Matos \& Lima-Costa, 2007). Esses dados remetem a uma maior facilidade de acesso aos serviços quando se tem um plano privado de saúde. 
Ainda com relação às características de facilitação para uso dos serviços de saúde bucal, no que se refere às características da comunidade, a região Sul foi referência para o uso há menos de um ano, sugerindo um uso mais recente entre os idosos dessa região. O uso há menos de um ano esteve associado também com residentes da zona urbana (Martins et al., 2008), como a maior chance de utilizar o serviço por rotina (Martins et al., 2008). Assim, infere-se que a acessibilidade aos serviços da zona urbana é facilitada quando comparada à zona rural. Publicações recentes enfatizam a relação da dificuldade de acesso aos serviços de saúde bucal por idosos residentes de comunidades rurais e/ou quilombolas, que são associadas à restrição local da oferta de serviço e à necessidade de planejamentos em saúde para a melhoria de políticas direcionadas a essa população (Miranda et al., 2020; Schroeder et al., 2020).

Ao identificar os fatores de necessidade de uso dos serviços de saúde, sintomas como dor dentária ou em gengivas esteve associada ao uso recente há menos de um ano (Sória et al., 2019; Souza et al., 2012; Martins et al., 2008; Martins et al., 2007). O uso por rotina foi associado com ausência de dor (Martins et al., 2008). Os fatores de diagnóstico de condições normativas de saúde bucal estiveram associados ao uso dos serviços de saúde bucal (Sória et al., 2019; Silva et al., 2018; Prado et al., 2018; Fonseca et al., 2017; Silva; Langlois \& Feldens, 2013; Ferreira et al., 2013; Martins et al., 2008; Martins et al., 2008; Martins et al., 2007). Sangramento gengival, presença de cálculo, bolsa periodontal e edentulismo foram associados ao uso há mais de um ano (Prado et al., 2018; Silva et al., 2013). A condição periodontal saudável, não ter necessidade de próteses, de tratamento odontológico e menor número de dentes extraídos estiveram associados ao uso recente (Sória et al., 2019; Fonseca et al., 2017; Ferreira et al., 2013; Martins et al., 2008; Martins et al., 2007), regular (Silva et al., 2018) ou por rotina (Martins et al., 2008) dos serviços de saúde bucal. O fato de idosos terem saúde bucal promove o uso recente dos serviços. Aqueles sem dentes procuram menos os serviços. Isso pode ser explicado por não julgarem necessário (Veras, 2007) devido à ausência de dentes ou por não saberem da necessidade de reabilitação protética e ainda por ser um serviço com pouca cobertura no Brasil (Martins et al., 2008). A perda dentária, problema de saúde pública, reflete a ineficiência de ações preventivas e/ou curativas (Gilbert et al., 2003; Susin et al., 2005), sendo um importante indicador de saúde bucal. As práticas curativas e mutiladoras marcaram o modelo assistencial no Brasil (Sampaio \& Cruz, 2014). O edentulismo é um desafio para a saúde pública. Ele acarreta importantes prejuízos na vida das pessoas (Gilbert et al., 2003; Barbato et al., 2007; Polzer et al., 2012). As perdas dentárias evidenciam desigualdades sociais (Gilbert et al., 2003; Susin et al., 2005; Bernabé \& Marcenes, 2011; Peres et al., 2010), uma vez que condições econômicas são comumente causas diretas ou indiretas da perda dentária (Gilbert et al., 2003).

Ainda com relação às características da 'necessidade de uso', o uso dos serviços públicos de saúde bucal foi motivado pela dor ou extração dentária (Fonseca et al., 2017) e por sangramento, cavidade ou dor (Oliveira et al., 2016). Tais associações sugerem procura por motivos de urgência odontológica e não por rotina. Nessa perspectiva, estudo com idosos em Campina Grande, Paraíba, demonstrou baixa procura por rotina (17\%) (Xavier et al., 2013). Estudos sugerem que idosos brasileiros não utilizam os serviços odontológicos com a frequência recomendada (Silva et al., 2013; Ferreira et al., 2013; Pinheiro \& Torres, 2006). Diferentemente dos países desenvolvidos, como no Canadá, em que $75 \%$ dos idosos entrevistados tinham consultado um dentista no ano anterior e 6\% deles com urgência (Ramraj et al., 2012). Também, nos EUA, Dados do National Health Interview Survey (NHIS), de 2015, apontaram prevalência de 62\% de consulta ao dentista no último ano (National Health Interview Survey, 2015). No Reino Unido, estudo revelou que 38\% dos participantes compareceram regularmente ao dentista, apesar de que 44\% deles foram por motivos de urgência (Kassim \& Croucher, 2012).

Revisão sistemática e metanálise, conduzida no Brasil, em 2017, estimou 37\% de prevalência da utilização de serviços de saúde bucal no último ano, para todas as idades (Araújo et al., 2017). Mesmo não havendo uma evidência sobre a periodicidade ideal para se procurar o dentista (Clarkson \& Worthington, 2007), Araújo et al. (2017) revelaram que a maior 
parte da população não procura o serviço a cada seis meses, segundo a orientação tradicional. A baixa prevalência de uso dos serviços reflete diretamente no quadro negativo de saúde bucal da população brasileira.

A atual revisão apresentou associação entre percepção da condição de saúde e uso dos serviços, em sete estudos (Fonseca et al., 2017; Oliveira et al., 2016; Silva et al., 2013; Martins et al., 2008; Martins et al., 2008; Martins et al., 2007; Matos \& Lima-Costa, 2007). A autopercepção negativa foi predominante para aqueles que usaram o serviço no último ano. Idosos dentados perceberam sua fala prejudicada em função da condição bucal e a aparência afetada por problemas na boca ou dentes (Martins et al., 2008) e perceberam a mastigação ruim/ péssima (Martins et al., 2007). O uso dos serviços públicos de saúde bucal foi maior entre aqueles com autopercepção negativa de sua condição bucal e a aparência (Fonseca et al., 2017), além de perceberem o relacionamento afetado por problemas na boca/dentes (Oliveira et al., 2016). O uso rotineiro foi associado com a autopercepção ótima/boa da mastigação entre dentados e com relacionamento não afetado, entre dentados e edentados (Martins et al., 2008) Sabe-se que crenças e valores pessoais podem interferir na autopercepção da saúde geral e bucal por idosos e que barreiras no acesso podem desencorajar a procura dos serviços (Martins et al., 2014; Benedetti et al., 2007). Idosos de Florianópolis, quando utilizaram o serviço público de saúde, queixaram-se do acesso com relação à marcação, à espera do atendimento e à falta de profissionais cirurgiões-dentistas (Benedetti et al., 2007).

Nesse sentido, considerando o quadro de aumento da expectativa de vida do Brasil e o envelhecimento que traz demandas sociais e econômicas em todo o mundo, há que se ter políticas destinadas aos idosos. Políticas que reconheçam o idoso como recurso importante para a sociedade e que incentivem a prevenção, o cuidado e a atenção integral à saúde, inclusive da saúde bucal (Brasil, 2005; Sampaio \& Cruz, 2009). É sabido que a política Brasil Sorridente (Brasil, 2004) tem ampliado o acesso aos serviços. Todavia, pela precariedade das condições de saúde bucal e acesso reduzido dos idosos aos serviços de saúde ${ }^{10}$, recomenda-se que esse trabalho subsidie a implementação de tais políticas, dada a sumarização dos fatores associados ao uso dos serviços de saúde bucal pelos idosos brasileiros (Sória et al., 2019; Silva et al., 2018; Prado et al., 2018; Fonseca et al., 2017; Oliveira et al., 2016; Silva et al., 2013; Ferreira et al., 2013; Souza et al., 2012; Martins et al., 2008; Martins et al.,, 2008; Martins et al.,, 2007; Matos \& Lima-Costa, 2007).

Há uma heterogeneidade na exposição das variáveis desfecho relativas ao uso de serviços de saúde bucal. Quanto ao tempo da última consulta, as investigações analisaram uso no último ano, últimos dois anos, últimos três anos, uso regular, uso por rotina e tipo de serviços utilizados, além de nunca ter utilizado o serviço odontológico. Usar regularmente e usar rotineiramente são variáveis que carecem de melhor esclarecimento sobre o que se considerar como rotina e como regular. Dois estudos consideraram somente o uso dos serviços públicos, portanto, o menor uso desses serviços pode estar atrelado ao maior uso do privado, que também seria um indicador de uso de serviços de saúde bucal por idosos. Outra limitação é o fato da incorporação de estudos que analisaram banco de dados originados de uma mesma pesquisa, apesar de testar diferentes variáveis desfecho no modelo estatístico ou analisar subgrupos populacionais. Destaca-se, ainda, que os estudos avaliados são transversais, o que implica na impossibilidade de estabelecer causalidade entre as associações evidenciadas.

Destacam-se pontos positivos desta revisão. Não houve limitação na busca quanto ao ano de publicação, possibilitando incorporar um maior número de estudos. Além disso, todos os estudos adotaram a análise estatística múltipla, o que acentua a força da evidência dos fatores associados ao uso dos serviços. Recomenda-se buscar um consenso quanto ao melhor parâmetro de regularidade para as consultas odontológicas. Importante, também, definir o melhor recorte para avaliar tempo da última consulta, para uniformização em futuros estudos, a fim de facilitar a sumarização e discussão dos resultados, na busca da melhor evidência. Muitos foram os fatores associados, portanto sugerem-se futuras pesquisas, do tipo revisões sistemáticas com metanálise, a partir de subgrupos mapeados nesta Scoping Review. 


\section{Conclusão}

Os estudos apresentaram fatores associados ao uso de serviços de saúde bucal pelos idosos brasileiros, na perspectiva das características de predisposição, de facilitação e de necessidade de uso, ratificando o modelo teórico de Andersen e Newman. Os fatores com maior destaque nos estudos foram os sociodemográficos (escolaridade e renda) e de necessidade clínica. Diferentes fatores interferem no acesso dificultado aos serviços de saúde bucal pelo estrato etário idoso, gerando um quadro grave de doenças bucais, necessidade acumulada de tratamento e de reabilitação. A síntese e o mapeamento dos resultados dos estudos, realizados no Brasil, poderão estimular estratégias de implementação de políticas públicas, que ampliem a utilização de serviços de saúde bucal para idosos, a partir do enfrentamento de barreiras geradoras de desigualdades no acesso a esses serviços.

\section{Referências}

Anderson, S., Allen, P., Peckham, S., \& Goodwin, N. (2008). Asking the right questions: scoping studies in the commissioning of research on the organisation and delivery of health services. Health Research Policy and Systems, 6:12. https://www.ncbi.nlm.nih.gov/pmc/articles/PMC2500008/

Andersen, R. M., \& Davidson, P. L. (2007). Improving access to care in America. Changing the US health care system: key issues in health services policy and management. San Francisco: Jossey-Bass.

Andersen, R. M., \& Davidson, P. L. (1997). Ethnicity, aging, and oral health outcomes: a conceptual framework. Advances in Dental Research, 11(2): 203209. https://journals.sagepub.com/doi/abs/10.1177/08959374970110020201

Andersen, R. M. (1995). Revisiting the behavioral model and access to medical care: does it matter? Journal of Health and Social Behavior, 36(1):1-10. https://www.jstor.org/stable/2137284?seq=1

Andersen, R. M., \& Newman, J. F. (1973). Societal and individual determinants of medical care utilization in the United States. Milbank memorial fund quarterly, 51: 95-124. https://www.ncbi.nlm.nih.gov/pmc/articles/PMC2690261/

Araújo, M. E. A., Silva, M. T., Andrade, K. R. C., Galvão, T. F., \& Pereira, M. G. (2017). Prevalência de utilização de serviços de saúde no Brasil: revisão sistemática e metanálise. Epidemiologia e Serviços de Saúde, 26(3): 589-604. http://www.scielo.br/scielo.php?script=sci_arttext\&pid=S2237$96222017000300589 \& \operatorname{lng}=$ en

Arksey, H., \& O’Malley, L. (2005). Scoping studies: towards a methodological framework. International Journal of Social Research Methodology, 8:19-32. https://core.ac.uk/download/pdf/56237.pdf

Austregésilo, S. C., Leal, M. C. C., Marques, A. P. O., Vieira, J. C. M., \& Alencar, D. L. (2015). Acessibilidade a serviços de saúde bucal por pessoas idosas: uma revisão integrativa. Revista Brasileira de Geriatria e Gerontologia, 18(1):189-199. http://www.scielo.br/scielo.php?script=sci_arttext\&pid=S1809$98232015000100189 \& \operatorname{lng}=$ en

Baldani, M. H., Brito, V. H., Lawder, J. A. C., Mendes, Y. B. E., Silva, F. F. M., \& Antunes, J. L. F. (2010). Determinantes individuais da utilização de serviços odontológicos por adultos e idosos de baixa renda. Revista Brasileira de Epidemiologia, 13(1):150-162. http://www.scielo.br/scielo.php?script=sci_arttext\&pid=S1415790X2010000100014\&lng=en\&nrm=iso.

Barbato, P. R., Nagano, H. C. M., Zanchet, F. N., Boing, A. F., \& Peres, M. A. (2007). Perdas dentárias e fatores sociais, demográficos e de serviços associados em adultos brasileiros: uma análise dos dados do Estudo Epidemiológico Nacional (Projeto SB Brasil 2002-2003). Cadernos de Saúde Pública, 23(8): 1803-14. http://www.scielo.br/scielo.php?script=sci_arttext\&pid=S0102-311X2007000800007\&lng=en\&nrm=iso

Benedetti, T. R. B., Mello, A. L. S. F., \& Gonçalves, L. H. T. (2007). Idosos de Florianópolis: autopercepção das condições de saúde bucal e utilização de serviços odontológicos. Ciência \& Saúde Coletiva, 12(6):1683-90. http://www.scielo.br/scielo.php?script=sci_arttext\&pid=S1413$81232007000600029 \& \operatorname{lng}=\mathrm{en} \& \mathrm{nrm}=\mathrm{iso}$

Bernabé, E., \& Marcenes, W. (2011). Income inequality and tooth loss in the United States. Journal of Dental Research, 90(6): 724-729. https://journals.sagepub.com/doi/abs/10.1177/0022034511400081

Brasil. (2012). SB Brasil 2010: Pesquisa Nacional de Saúde Bucal: resultados principais. Ministério da Saúde, 2012. http://bvsms.saude.gov.br/bvs/publicacoes/pesquisa_nacional_saude_bucal.pdf Acesso em: 17 dez 2018.

Brasil. (2005). Envelhecimento ativo: uma política de saúde. da Saúde. http://bvsms.saude.gov.br/bvs/publicacoes/envelhecimento_ativo.pdf 8. Acesso em: 10 dez 2018 .

Brasil. Diretrizes da Política Nacional de Saúde Bucal. http://189.28.128.100/dab/docs/publicacoes/geral/diretrizes_da_politica_nacional_de_saude_bucal.pdf Acesso em: 07 jun 2019.

Brasil. (2004). Projeto SB Brasil 2003: Condições de saúde bucal da população brasileira 2002-2003: resultados principais. Ministério da Saúde, Secretaria de Atenção à Saúde, Departamento de Atenção Básica. - Brasília. https://bvsms.saude.gov.br/bvs/publicacoes/condicoes_saude_bucal.pdf

Clarkson, J. E., \& Worthington, H. V. (2007). Recall intervals for oral health in primary care patients. Cochrane database of Systematic Reviews, 17(4): CD004346. https://www.cochrane.org/CD004346/ORAL_recall-intervals-for-oral-health-in-primary-care-patients 
Davidson, P. L., \& Andersen, R. M. (1997). Determinants of dental care utilization for diverse ethnic and age groups. Advances in Dental Research, 11:254262. https://journals.sagepub.com/doi/abs/10.1177/08959374970110020801

Ferreira, C. O., Antunes, J. L. F., \& Andrade, F. B. (2013). Factors associated with the use of dental services by elderly Brazilians. Revista de Saúde Pública, 47(Suppl 3): 90-97. http://www.scielo.br/scielo.php?script= sci_arttext\&pid=S0034-89102013000900090\&lng=en\&nrm=iso

Fonseca, E. P., Fonseca, S. G. O., \& Meneghim, M. C. (2017). Factors associated with the use of dental care by elderly residents of the state of São Paulo, Brazil. Revista Brasileira de Geriatria e Gerontologia, 20(6):785-796. http://www.scielo.br/scielo.php?script=sci_arttext\&pid=S180998232017000600785\&lng=en.

Gilbert, G. H., Duncan, R. P., \& Shelton, B. J. (2003). Social Determinants of Tooth Loss. Health Services Research, 38:1843-1862. https://www.ncbi.nlm.nih.gov/pmc/articles/PMC1360976/

Godfrey, C., Harrison, M., Lang, A., Macdonald, M., Leung, T., \& Swab, M. (2013). Homecare safety and medication management with older adults: a scoping review of the quantitative and qualitative evidence. Systematic Reviews and Implementation Reports, 11:82-130. https://www.queensu.ca/qjbc/sites/webpublish.queensu.ca.qjbcwww/files/files/Godfrey\%20Harrison\%20et\%20al\%202013\%20-\%20Homecare\%20safety.pdf

Holm-Pedersen, P., Vigild, M., Nitschke, I., \& Berkey, D. B. (2005). Dental care for aging populations in Denmark, Sweden, Norway, United kingdom, and Germany. Journal of Dental Education, 69:987-997. http://www.jdentaled.org/content/69/9/987.long

Kassim, S., \& Croucher, R. (2012). Factors associated with dental and medical care attendance in UK resident Yemeni khat chewers: a cross sectional study. BMC Public Health, 12: 486. https://www.ncbi.nlm.nih.gov/pmc/articles/PMC3598685

Listl, S. (2011). Income-related inequalities in dental service utilization by Europeans aged 50+. Journal of Dental Research, 90(6): 717-723. https://www.ncbi.nlm.nih.gov/pmc/articles/PMC3318029/

Machado, L. P., Camargo, M. B. J., Jeronymo, J. C. M., \& Bastos, G. A. N. (2012). Uso regular de serviços odontológicos entre adultos e idosos em região vulnerável no sul do Brasil. Revista de Saúde Pública, 46(3): 526-533. http://www.scielo.br/scielo.php?script=sci_arttext\&pid=S0034$89102012000300015 \& \operatorname{lng}=$ en.

Malta, D. C., \& Silva Jr, J. B. (2013). O Plano de Ações Estratégicas para o Enfrentamento das Doenças Crônicas Não Transmissíveis no Brasil e a definição das metas globais para o enfrentamento dessas doenças até 2025: uma revisão. Revista Epidemiologia e Serviços de Saúde, 22(1):151-164. http://scielo.iec.gov.br/scielo.php?script=sci_arttext\&pid=S1679-49742013000100016\&lng=es\&nrm=iso

Mariño, R. J., Khan, A. R., Tham, R., Khew, C-W., \& Stevenson, C. (2014) Pattern and factors associated with utilization of dental services among older adults in rural Victoria. Australian Dental Journal, 59(4): 504-510. https://pubmed.ncbi.nlm.nih.gov/25131698/

Martins, A. M. E. B. L., Jardim, L. A. S., Souza, J. G. S., Rodrigues, C. A. Q., Ferreira, R. C., \& Pordeus, I. A. (2014). Is the negative evaluation of dental services among the Brazilian elderly population associated with the type of service? Revista Brasileira de Epidemiologia, 17(1):71-90. http://www.scielo.br/scielo.php?script=sci_arttext\&pid=S1415-790X2014000100071\&lng=en\&nrm=iso

Martins, A. M. E. B. L., Haikal, D. S., Pereira, S. M., \& Barreto, S. M. (2008). Routine use of dental services by the elderly in Brazil: the SB Brazil Project. Cadernos de Saúde Pública. 24(7):1651-1666. http://www.scielo.br/scielo.php?script=sci_arttext\&pid=S0102-311X2008000700020\&lng=en\&nrm=iso

Martins, A. M. E. B. L., Barreto, S. M., \& Pordeus, I. A. (2008). Características associadas ao uso de serviços odontológicos entre idosos dentados e edentados no Sudeste do Brasil: Projeto SB Brasil. Cadernos de Saúde Pública,24(1):81-92. http://www.scielo.br/scielo.php?script=sci_arttext\&pid=S0102$311 X 2008000100008 \& \operatorname{lng}=$ en $\& n r m=$ iso

Martins, A. M. E. B. L., Barreto, S. M., \& Pordeus, I. A. (2007). Uso de serviços odontológicos entre idosos brasileiros. Revista Panamericana de Salud Pública, 22(5): 308-16. http://www.scielo.br/scielo.php?script=sci_arttext\&pid=S0102-311X2008000700020\&lng=en\&nrm=iso

Matos, D. L., \& Lima-Costa, M. F. (2007). Tendência na utilização de serviços odontológicos entre idosos brasileiros e fatores associados: um estudo baseado na Pesquisa Nacional por Amostra de Domicílios $(1998$ e 2003). Cadernos de Saúde Pública, 23(11):2740-2748. http://www.scielo.br/scielo.php?script=sci_arttext\&pid=S0102-311X2007001100021\&lng=en\&nrm=iso

Mendes, E. V. (2012). O cuidado das condições crônicas na atenção primária à saúde: o imperativo da consolidação da estratégia da saúde da família. Brasília: Organização Pan-Americana da Saúde. http://bvsms.saude.gov.br/bvs/publicacoes/cuidado_condicoes_atencao_primaria_saude.pdf Acesso em: 10 $\operatorname{dez} 2018$.

Miranda, G. M. D., Mendes, A. C. G., \& da Silva, A. L. A. (2016). O envelhecimento populacional brasileiro: desafios e consequências sociais atuais e futuras. Revista Brasileira de Geriatria e Gerontologia, 19(3): 507-519.

Miranda, L. P., Oliveira, T. L., Queiroz, P. S. F., Oliveira, P. S. D., Fagundes, L. S., \& Neto, J. F. R. (2020). Saúde bucal e acesso aos serviços odontológicos em idosos quilombolas: um estudo de base populacional. Revista Brasileira de Geriatria e Gerontologia, 23(2): e200146. https://www.scielo.br/j/rbgg/a/9Kh9YPZ6NTwmzbLJJdbMpgr/?format=pdf\&lang=pt

Moher, D., Liberati, A., Tetzlaff, J., Altman, D. G., \& Group, P. (2009). Preferred reporting items for systematic reviews and meta-analyses: the PRISMA statement. PLoS Medicine, 6(7): e1000097. https://www.ncbi.nlm.nih.gov/pmc/articles/PMC2707599/pdf/pmed.1000097.pdf

Moreira, R. S., Nico, L. S., Tomita, N. E., \& Ruiz, T. (2005). A saúde bucal do idoso brasileiro: revisão sistemática sobre o quadro epidemiológico e acesso aos serviços de saúde bucal. Cadernos de Saúde Pública. 21(6):1665-1675. http://www.scielo.br/pdf/csp/v21n6/03.pdf

National Center for Health Statistics - NCHS. (2016). Health, United States, 2015: with special feature on racial and ethnic health disparities. Washington. http://www.cdc.gov/nchs/data/hus/hus15.pdf Acesso em: 16 dez 2019. 
Oliveira, R. F. R., Souza, J. G. S. Haikal, D. S'A., Ferreira, E. F., \& Martins, A. M. E. B. L. (2016). Equity in the use of dental services provided by the Brazilian Unified Health System (SUS) among the elderly: a population-based study. Ciência \& Saúde Coletiva, 21(11): 3509-3523. http://www.scielo.br/scielo.php?script=sci_arttext\&pid=S1413-81232016001103509\&lng=en\&nrm=iso

Peres, M. A., Barbato, P. R., Reis, S. C. G. B., Freitas, C. H. S. M., \& Antunes, J. L. F. (2013). Tooth loss in Brazil: analysis of the 2010 Brazilian Oral Health Survey. Revista de Saúde Pública, 47(Supl 3):1-11. http://www.scielo.br/scielo.php?script=sci_arttext\&pid=S0034-89102013000900078\&lng=en\&nrm=iso

Peters, M. D. J., Godfrey, C. M., Khalil, H., McInerney, P., Parker, D., \& Soares, C. B. (2015). Methodology for JBI scoping reviews. The Joanna Briggs Institute Reviewers' Manual 2015. Adelaide, South Australia: The Joanna Briggs Institute, 2015. http://joannabriggs.org/assets/docs/sumari/ReviewersManual_Methodology-for-JBI-Scoping-Reviews_2015_v2.pdf Acesso em: 10 jan 2019.

Peters, M. D. J., Godfrey, C. M., Khalil, H., McInerney, P., Parker, D., \& Soares, C. B. (2015). Guidance for conducting systematic scoping reviews, Journal of Evidence-Based Healthcare, 13(3): 141-146. https://insights.ovid.com/article/01787381-201509000-00005

Pinheiro, R. S., \& Torres, T. Z. G. (2006). Uso de serviços odontológicos entre os Estados do Brasil. Ciência \& Saúde Coletiva, 11(4): 999-1010. http://www.scielo.br/scielo.php?script=sci_arttext\&pid=S1413-81232006000400021\&lng=en\&nrm=iso

Pinto, R. S., Matos, D. L., \& Loyola Filho, A. I. (2012). Características associadas ao uso de serviços odontológicos públicos pela população adulta brasileira. . Ciência \& Saúde Coletiva, 17(2): 531-544. https://www.scielosp.org/pdf/csc/2012.v17n2/531-544/pt

Polzer, I., Schwahn, C., Völzke, H., Mundt, T., \& Biffar, R. (2012). The association of tooth loss with all-cause and circulatory mortality. Is there a benefit of replaced teeth? A systematic review and meta-analysis. Clinical Oral Investigations, 16:333-51. https://link.springer.com/article/10.1007\%2Fs00784-011$0625-9$

Prado, R. L., Garbin, C. A. S., Moimaz, S. A. S., \& Saliba, N. A. (2018). Inequalities in Oral Health among Older Brazilians: A Cross-Sectional Analysis of a National Survey. Pesquisa Brasileira em Odontopediatria e Clínica Integrada, 18(1): e3726. http://revista.uepb.edu.br/index.php/pboci/article/view/10/pdf

Ramraj, C., Azarpazhooh, A., Dempster, L., Ravaghi, V., \& Quiñonez, C. (2012). Dental treatment needs in the Canadian population: analysis of a nationwide cross-sectional survey. BMC Oral Health, 12: 46. https://www.ncbi.nlm.nih.gov/pmc/articles/PMC3508863/

Sampaio, D. M. L., \& Cruz, L. M. F. S. (2014). Perfil de utilização dos serviços odontológicos públicos e privados pela população adulta brasileira. Revista Brasileira de Pesquisa em Saúde, 16(3):14-22. https://www.scielo.br/j/csc/a/G6MFqJKjFRqH4h3379TgYMb/?format=pdf\&lang=pt

Schroeder, F. M. M., Mendoza-Sassi, R. A., \& Meucci, R. D. (2020). Condição de saúde bucal e utilização de serviços odontológicos entre idosos em área rural no sul do Brasil. Ciência \& Saúde Coletiva, 25(6): 2093-2102. https://www.scielo.br/j/csc/a/wzbYR5sTqsHNYMgvrkbfmZb/?format=pdf\&lang=pt

Silva, A.E.R., Echeverria, M. S., Custódio, N. B., Cascaes, A. M., Camargo, M. B. J., \& Langlois, C. O. (2018). Regular use of dental services and dental loss among the elderly. Ciência \& Saúde Coletiva, 23(12):4269-4276. http://www.scielo.br/scielo.php?script=sci_arttext\&pid=S1413-81232018001204 $269 \& \operatorname{lng}=$ en\&nrm=iso

Silva, A. E. R., Langlois, C. O., \& Feldens, C. A. (2013). Use of dental services and associated factors among elderly in southern Brazil. Revista Brasileira de Epidemiologia, 16(4): 1005-1016. http://www.scielo.br/scielo.php?script=sci_arttext\&pid=S1415-790X2013000401005\&lng=en\&nrm=iso

Sória, G. S., Nunes, B. P., Bavaresco, C. S., Vieira, L. S., Facchini, L. A. (2019). Acesso e utilização dos serviços de saúde bucal por idosos de Pelotas, Rio Grande do Sul, Brasil. Cadernos de Saúde Pública, 35(4):e00191718. http://www.scielo.br/scielo.php?script=sci_arttext\&pid=S0102311 X2019000405011\&lng=pt\&nrm=iso

Souza, E. H. A., Oliveira, P. A. P., Paegle, A. C., \& Goes, P. S. A. (2012). Raça e o uso dos serviços de saúde bucal por idosos. Ciência \& Saúde Coletiva, 17(8): 2063-2070. http://www.scielo.br/scielo.php?script=sci_arttext\&pid=S1413-81232012000800017\&lng=en\&nrm=iso

Stopa, S. R., Malta, D. C., Monteiro, C. N., Szwarcwald, C. L., Goldbaum, M., \& Cesar, C. L. G. (2017). Acesso e uso de serviços de saúde pela população brasileira, Pesquisa Nacional de Saúde 2013. Revista de Saúde Pública, 51(Suppl 1):3s. http://www.scielo.br/scielo.php?script=sci_arttext\& pid=S0034$89102017000200308 \& \operatorname{lng}=$ en

Susin, C., Oppermann, R. V., Haugejorden, O., \& Albandar, J. M. (2005). Tooth loss and associated risk indicators in an adult urban population from south Brazil. Acta Odontologica Scandinavica, 63:85-93. https://www.tandfonline.com/doi/abs/10.1080/00016350510019694?journalCode=iode20

Tsakos, G., Sabbah, W., Chandola, T., Newton, T., Kawachi, I., Aida, J, et al. (2013). Social relationships and oral health among adults aged 60 years or older. Psychosomatic Medicine, 75(2): 178-186. https://pubmed.ncbi.nlm.nih.gov/23324876/

Veras, R. (2009). Envelhecimento populacional contemporâneo: demandas, desafios e inovações. Revista de Saúde Pública, 43(3):548-554. http://www.scielo.br/scielo.php?script=sci_arttext\&pid=S0034-89102009000300020\&lng=en\&nrm=iso

Xavier, A. F. C., Santos, J. A., Alencar, C. R. B., Andrade, F. J. P., Clementino, M. A., Menezes, T. N. et al. (2013). Uso dos serviços odontológicos entre idosos residentes no município de Campina Grande, Paraíba. Pesquisa Brasileira em Odontopediatria e Clínica Integrada, 13(4):371-76. http://revista.uepb.edu.br/index.php/pboci/article/viewFile/2251/pdf_21 